\title{
Radiation induces epithelial to mesenchymal transition via upregulation of PD-L1 in nasopharyngeal carcinoma cell
}

\author{
Heming Li ${ }^{1,2 \#} \wedge$, Zhi Wang ${ }^{1,2 \#}$, Shanshan Liang ${ }^{1,2 \#}$, Qiuge Liu ${ }^{1,2}$, Piao Wang ${ }^{1,2}$, Longyu Cai $^{1}$, Ruoyu Wang ${ }^{1,2}$ \\ ${ }^{1}$ Department of Oncology, Affiliated Zhongshan Hospital of Dalian University, Dalian, China; ${ }^{2}$ The Key Laboratory of Biomarker High Throughput \\ Screening and Target Translation of Breast and Gastrointestinal Tumor, Dalian, China \\ Contributions: (I) Conception and design: H Li, Z Wang, S Liang, R Wang; (II) Administrative support: Z Wang, Q Liu; (III) Provision of study \\ materials: P Wang; (IV) Collection and assembly of data: L Cai; (V) Data analysis and interpretation: L Cai; (VI) Manuscript writing: All authors. (VII) \\ Final approval of manuscript: All authors. \\ "These authors contributed equally to this work. \\ Correspondence to: Ruoyu Wang. Department of Medical Oncology, Affiliated Zhongshan Hospital of Dalian University, No. 6 Jiefang Street, Dalian \\ 110006, China. Email: wangruoyu1963@hotmail.com.
}

Background: The acquisition of radioresistance by nasopharyngeal carcinoma (NPC) cells during radiotherapy may lead to tumor metastasis and poor survival. This study aimed to explore the mechanism of long-term radiation-induced NPC metastasis.

Methods: The radioresistant NPC cell, Hone-1R, was established for further study. A colony-forming assay was selected for the evaluation of radioresistant capacity, while a scratch wound healing assay was used to detect tumor cell migration. The expression of relative protein levels were detected by Western blot (WB) analysis and immunofluorescence. Cell morphology was acquired by microscopy. The programmed cell death ligand-1 (PD-L1) expression level in NPC tumor tissues was evaluated based on the publicly available datasets of NPC patients.

Results: A radioresistant NPC cell, Hone-1R, was established with a total dose of 180 Gy, and verified by radioresistant capacity testing. The morphology of Hone-1R cells showed obvious mesenchymal-like cells. WB and wound healing assays indicated that Hone-1R cells exhibited an epithelial-mesenchymal transition (EMT) phenotype with high migration ability and upregulation of PD-L1. Knockdown of PD-L1 reversed EMT status and reduced the migration ability of Hone-1R cells. Further analysis indicated that PD-L1 was overexpressed in more advanced stages and was positively correlated with the EMT score in NPC patients based on in silico analysis.

Conclusions: Our study revealed that long-term radiation induces EMT and increases migration ability of NPC radioresistant cells through upregulation of PD-L1. These results advance our investigation of the underlying mechanism of ionizing radiation (IR)-induced migration, and suggest potential interventions to reverse EMT-induced acquisition of radioresistance in NPC.

Keywords: Nasopharyngeal carcinoma (NPC); migration; radioresistance; programmed cell death ligand 1 (PDL1); epithelial-mesenchymal transition (EMT)

Submitted Apr 22, 2020. Accepted for publication Oct 28, 2020.

doi: $10.21037 /$ tcr-20-1899

View this article at: http://dx.doi.org/10.21037/tcr-20-1899

^ ORCID: 0000-0002-5702-0147. 


\section{Introduction}

Nasopharyngeal carcinoma (NPC) is a malignancy with high prevalence in the eastern and southeastern regions of Asia, and originates from the epithelium of the nasopharynx (1). Due to the complex anatomical structure of the nasopharynx, invasive properties of NPC cells, sensitivity to radiation, and other factors, radiotherapy is the primary therapeutic strategy for NPC $(2,3)$. With wide application of precision radiotherapy techniques, such as intensity-modulated radiotherapy and concurrent radiochemotherapy, the rate of local control has significantly improved. The 5 -year survival rate is close to $90 \%$ for early stage NPC patients (stage I and II). However, optimizing the efficacy of radiotherapy for late stage NPC patients (stage III and IV) is a challenge. Approximately $60-70 \%$ of locally advanced NPC patients are identified with a highrisk of treatment failure $(4,5)$. Radiation-induced metastasis is a key reason for the failure of therapy and poor prognosis. Therefore, exploring the underlying molecular mechanism and screening potential therapeutic targets have important clinical significance for improving outcomes for NPC patients.

Accumulating evidence has suggested that a few of the tumor cells undergoing epithelial-mesenchymal transition (EMT) will persist under radiation and produce metastatic lesions $(6,7)$. The phenomenon of EMT is appreciable, with epithelial cells transforming into mesenchymal cells under physiological and pathological conditions (8). Recently, numerous studies have implicated that long-term exposure to ionizing radiation (IR) initiates tumor cells to undergo an EMT process, which promotes radioresistance and metastasis $(9,10)$. However, the molecular mechanism remains unclear. Hence, exploring the potential mechanism is of great significance in the endeavor to reverse the resulting radioresistance and metastasis in NPC.

A number of studies have proven that programmed cell death ligand-1 (PD-L1), also known as B7-H1 or CD274, is overexpressed in various types of tumor cells and is associated with different clinical outcomes including NPC $(11,12)$. Expression of PD-L1 interacts with its receptor, PD-1, and induces immune surveillance escape, which promotes tumor development and metastasis (13). A very recent study reported that $\mathrm{PD}-\mathrm{L} 1$ could induce EMT and promote tumor invasion in NPC cells without IR stimulation (14); however, the action of PD-L1 in a longterm exposure radiation-induced NPC radioresistant cell model is not clear. Thus, in our study, a radioresistant NPC cell line (Hone-1R) was established by exposure to incrementally increasing doses of IR in the Hone- 1 cell line. We sought to evaluate PD-L1-induced EMT status in radioresistance and migration in the Hone- $1 \mathrm{R}$ cell line to provide new insights into the development of a therapy for radioresistant NPC.

We present the following article in accordance with the MDAR checklist (available at http://dx.doi.org/10.21037/ tcr-20-1899).

\section{Methods}

\section{Materials and reagents}

The human NPC cell line Hone-1 was received as a generous gift from the Department of Radiation Oncology, Sun Yat-sen University Cancer Center (Guangzhou, Guangdong, China). The jetPRIME ${ }^{\circledR}$ transfection reagent was purchased from Polyplus-transfection (Strasbourg, France). Antibodies to E-cadherin (Cell Signaling Technology (CST), Cat\# 3195, RRID:AB_2291471), vimentin (CST, Cat.\# 5741, RRID:AB_ 10695459), PDL1 (CST, Cat.\# 13684, RRID:AB_2687655), ZO-1 (CST, Cat.\# 8193, RRID:AB_10898025), N-cad (CST, Cat.\# 13116, RRID:AB_2687616), $\beta$-Catenin (CST, Cat.\# 8480, RRID:AB_11127855), and GAPDH (CST, Cat.\# 5174, RRID:AB_10622025) were purchased from CST, Inc. (Beverly, MA, USA). Anti-ZEB-2 (Santa Cruz Biotechnology (SCBT), Cat.\# sc-271984, RRID: AB_10708399), secondary goat anti-rabbit (SCBT, Cat.\# sc2004, RRID:AB_631746), and goat anti-mouse antibodies (SCBT, Cat.\# sc-2005, RRID:AB_631736) were purchased from SCBT, Inc. (Santa Cruz, CA, USA).

\section{Cell culture and the establishment of radioresistant Hone-1R cell}

The Hone-1 cell line was cultured in 1640 medium containing $10 \%$ fetal bovine serum (FBS) at $37{ }^{\circ} \mathrm{C}$ with $5 \% \mathrm{CO}_{2}$. The cells were passaged every $2-3$ days. The radioresistant cell line Hone-1R was established according to previous publications. The cell line Hone- 1 was seeded at a density of $3 \times 10^{5}$ cells per $6 \mathrm{~cm}$ dish. The cells were initially subjected to 2 Gy of IR when the status was stable. The Hone- 1 cells were cultured and passaged twice after the initial radiation, and then were exposed to another 2 Gy of IR. The surviving cells were then treated in accordance with the above methods and subjected to a series of gradually increasing doses of IR exposure (4, 6, 8, and 10 Gy) twice. After 60 Gy of total IR doses, the cells began 
to receive 8 Gy of IR exposure each time. When the total IR dose reached 180 Gy, IR exposure was terminated. The final surviving cells were tested with a series of radiosensitivity capacity experiments, verified as radioresistant NPC cells, and named as Hone-1R. The parental control cells and Hone-1 cells were treated with the same procedure but without the IR exposure. The cells were cultured within 5-10 passages after the termination of the final IR exposure for further experiments. The entire establishment procedure lasted $>10$ months.

\section{Western blot (WB) assay}

Cells were rinsed 3 times with $4^{\circ} \mathrm{C} 1 \times$ phosphate-buffered saline (PBS) and placed on ice. Total protein was extracted using CelLytic MT cell lysis reagent (Sigma-Aldrich, St. Louis, MO, USA). The protein quantification was measured by Broadford test kit according to the manufacturer's instructions (Sangon Biotech, Shanghai, China). All the proteins were equally matched for quality and mixed with $5 \times$ loading buffer, and were then boiled in $100{ }^{\circ} \mathrm{C}$ for $5 \mathrm{~min}$. For the WB assay, equal amounts of protein samples were separated by $10 \%$ SDS-polypropylene gel and transferred to $0.2 \mu \mathrm{m}$ polyvinylidene difluoride (PVDF) membrane (Millipore, Billerica, MA, USA). Blocking was performed in $5 \%$ skimmed milk at room temperature (RT) for $1 \mathrm{~h}$, and the corresponding molecular weight proteins were cut. Next, the membranes were washed 3 times with $1 \times$ trisbuffered saline with Tween 20 (TBST) (Sangon Biotech, Shanghai, China), and then added to a primary antibody at $4{ }^{\circ} \mathrm{C}$ overnight. The next day, the membranes were washed with $1 \times$ TBST 3 times at RT. Secondary antibodies were incubated for $30 \mathrm{~min}$ at RT. After washing with $1 \times$ TBST, proteins were visualized by WesternBright ${ }^{\mathrm{TM}}$ enhanced chemiluminescence (ECL) (Advansta Inc., San Jose, CA, USA).

\section{Colony forming assay}

Different cell densities (200, 200, 400, 800, 1,200, and 1,600 per well) were seeded in 6 -well plates for $12 \mathrm{~h}$ and the cells were exposed to IR with increasing doses (2, 4, 6, 8, 10 Gy). The cells were then cultured for 10 days at $37{ }^{\circ} \mathrm{C}$ with $5 \% \mathrm{CO}_{2}$ in an incubator and the surviving colonies were calculated $(>50$ cells were considered as a colony). The cells were fixed with $4 \%$ paraformaldehyde (Beyotime, Shanghai, China) for $15 \mathrm{~min}$ and stained with $0.1 \%$ crystal violet (Solarbio, Beijing, China). Plating efficiencies (PE) were calculated as the number of colonies divided by the number of cells seeded. The survival fractions ( $\mathrm{SF}$ ) were calculated as $\mathrm{PE}$ divided by the control group that was not exposed to radiation (0 Gy). The experiment was repeated 3 times. A multi-target click model was used to fit cell survival curves $\left\{\mathrm{y}=1-\left[1-\exp \left(-\mathrm{k}^{*} \mathrm{x}\right)\right]^{\wedge} \mathrm{N}\right\}$ by GraphPad Prism 7 software (GraphPad Software, La Jolla, CA, USA). The software SPSS version 20.0 (IBM, Chicago, IL, USA) was used to analyze statistical differences. Statistical significance was set at $\mathrm{P}<0.05$.

\section{Scratch wound healing assay}

The in vitro wound healing assay is a convenient and economical method to assess cancer cell migration under EMT conditions (15). The cells were seeded in 6 -well culture plates. After $24 \mathrm{~h}$ of growth, the confluent monolayer of the cells were scratched with a $200 \mu \mathrm{L}$ pipette tip. The cells were then immediately washed twice with $1 \times$ PBS and incubated in serum-free 1640 media for $24 \mathrm{~h}$ and $48 \mathrm{~h}$. Photos were taken at different time points (0, 24, and 48 h). ImageJ software (Rasband, W.S., ImageJ, US National Institutes of Health, Bethesda, MD, USA) was used to analyze the wound area and calculate the migration rates. The migration rate was calculated as the experimental group migration area minus the control group migration area and then divided by the control group. The experiment was repeated 3 times and statistical analysis was conducted.

\section{Transient PD-L1 knockdown}

The cells were seeded at a density of $2.5 \times 10^{5}$ cells/ well in 6-well plates. The cells were transiently transfected with PD-L1 small interfering RNA (siRNA) or the negative control (siNC) using the jetPRIME ${ }^{\circledR}$ transfection agent according to the manufacturer's instructions. The PD-L1 siRNA sequence was 5'-CCAGCACACUGAGAAUCAATT3 ' ( s e n s e ), 5 ' - U U G A U U C U C A G U G U GCUGGTT-3'(anti-sense). The control sequence w a s U U C U C C G A A C G U G U C A C G U T T ACGUGACACGUUCGGAGAATT. Evaluation of WB analysis was undertaken to detect gene-silencing efficiency.

\section{Immunofluorescence}

The cells were seeded in cover slips for $48 \mathrm{~h}$. The glasses were taken out, placed in 6-well plates, and washed 3 times with $1 \times$ PBS. The cells were then fixed with $4 \%$ paraformaldehyde for $15 \mathrm{~min}$. After blocking with $1 \%$ FBS in PBS for $1 \mathrm{~h}$ at RT, the cells were incubated with the primary 
antibody PD-L1 (CST, Cat. \#86744, RRID: AB_2800088) at $4{ }^{\circ} \mathrm{C}$ overnight. After washing with $1 \times$ PBS 3 times, the cells were further incubated with a fluorescent secondary antibody (488, CST, Cat. \#4412, RRID:AB_1904025) for $1 \mathrm{~h}$ at RT. The cells were then incubated in 4',6-diamidino2-phenylindole (DAPI) for nucleus staining after washing 3 times with $1 \times$ PBS. Finally, immunofluorescent images were captured and analyzed by confocal acquisition software FV10-ASW 3.0 (Olympus Fluoview FV10-ASW, RRID: SCR_014215; Olympus, Tokyo, Japan).

\section{In silico analysis of PD-L1 in GSE103611}

Raw data from publicly available datasets, with focus on the largest dataset of NPC patients were downloaded from Gene Expression Omnibus (GEO) using accession number GSE103611 (PMID: 29428165). Gene set variation analysis (GSVA) (PMID: 23323831) was performed to calculate the enrichment score of mesenchymal-phenotype gene signatures for each patient in GSE103611. The signatures with names including "Mes-phenotype" were selected, and correlation tests were applied to determine the relationship between PD-L1 levels and the activation scores of the mesenchymal-related signatures. To further investigate potential functions and pathways associated with PD-L1 in NPC patients, gene set enrichment analysis (GSEA) was performed using R package "fgsea" in Bioconductor from the following site: http://www.ncbi. nlm.nih.gov/geo/query/acc.cgi? acc=GSE103611 (PMID: 16199517). The gene sets of cancer hallmarks from the Molecular Signatures Database (MSigDB) were used for running GSEA, and 1,000 permutations were used to calculate the $\mathrm{P}$ value.

\section{Statistical analysis}

The quantitative data are presented as the mean \pm standard deviation (SD) or standard error of mean (SEM) of three independent assays. Statistical analysis was performed using the unpaired Student's $t$-test ( $t$-test) and analysis of variance (ANOVA). The SPSS 20.0 software (RRID: SCR_002865) was used to analyze the data, with $\mathrm{P}<0.05$ indicating statistical significance.

\section{Ethical statement}

The study was conducted in accordance with the Declaration of Helsinki (as revised in 2013).

\section{Results}

\section{$A$ radioresistant Hone-1R cell line was established and the radioresistant capacity was validated}

The radioresistant NPC cell line (Hone-1R) was initially established by exposure to gradually increasing doses of IR in the Hone-1 cell line (Figure 1A). The colony-forming assay showed that the SF of Hone-1 cells receiving 60 or 100 Gy total doses of IR did not increase compared with that of the control group (Figure 1B,C). Thus, the survival cells continued to receive 8 Gy doses of IR exposure until the total exposure IR dose was 180 Gy. It was shown that greater numbers of cell colonies survived than those of their parental Hone-1 cell (Figure 1D). Further analysis showed that the SF of these surviving Hone-1 cells following incrementally increasing doses of IR grew significantly higher than that of their parental Hone-1 cell line $(\mathrm{P}=0.007$, Figure $1 E)$. Therefore, the radioresistant Hone-1 cell had been established successfully and was named the Hone-1R cell. Next, these cells were cultured over 30 passages in the absence of IR treatment for further experimentation.

\section{The radioresistant Hone-1R cell line presented an EMT- like phenotype with PD-L1 upregulation}

Dramatic morphological change occurred after Hone1 acquired radioresistance (Figure $2 A$ ). Most of the Hone-1R cells lost their tight cell-cell junctions, became scattered, and elongated with a mesenchymal cell-shaped feature. To evaluate the migration ability of the Hone-1R cell line, the scratch wound healing assay was performed. As shown in Figure 2B, the edge of the wound was neat following scratching in both the Hone-1 and Hone-1R groups. After $24 \mathrm{~h}$ of incubation, the cells migrated to the scratched area and the wound size gradually decreased. The migration rate of Hone-1R cells was obviously higher than that of the parental Hone-1 cells $(48 \%$ vs. $33 \%, \mathrm{P}=0.007$, Figure $2 B$ ).

The mechanism of EMT is crucial in contributing to the migration of tumor cells. To elucidate whether accumulating IR stimulation induces EMT and migration of radioresistant NPC cells, we examined EMT phenotypic markers by WB assay. As shown in Figure $2 C$, the epithelial markers E-cadherin and zonula occludens (ZO)-1 were downregulated in Hone-1R cells; mesenchymal markers were upregulated, including $\beta$-catenin, vimentin, ZEB-2, and $\mathrm{N}$-cadherin. Most importantly, the expression of PD- 
A

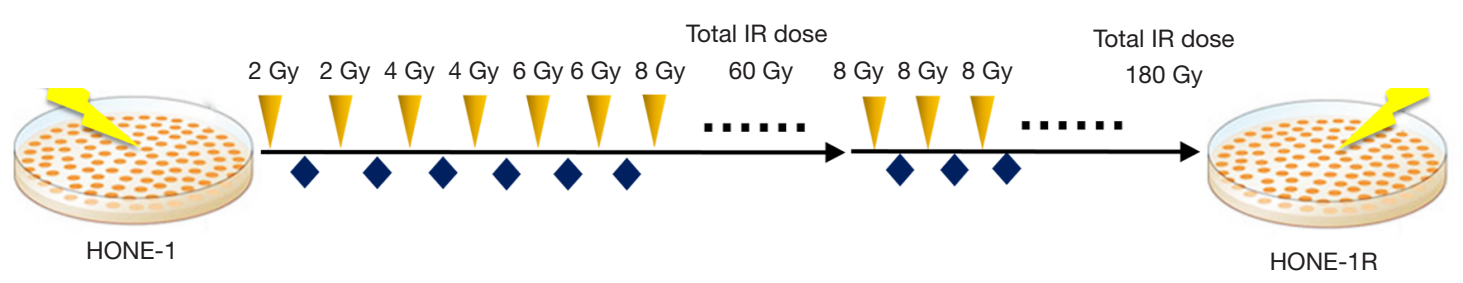

B

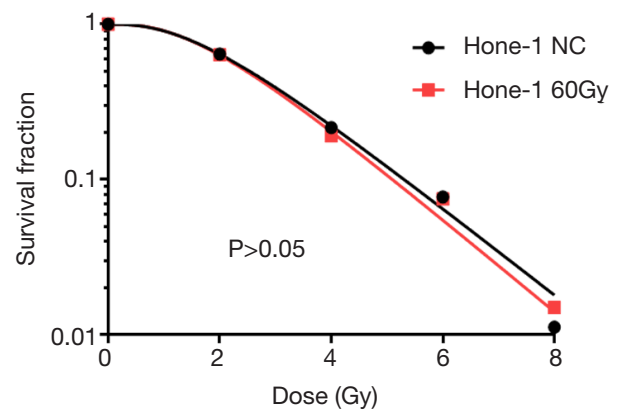

D
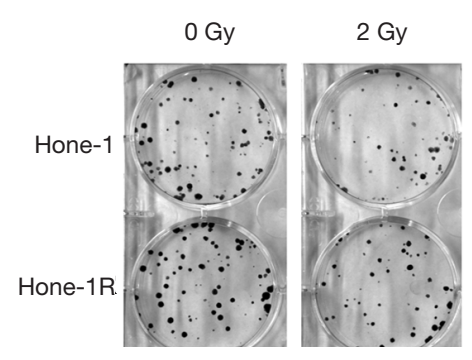

C

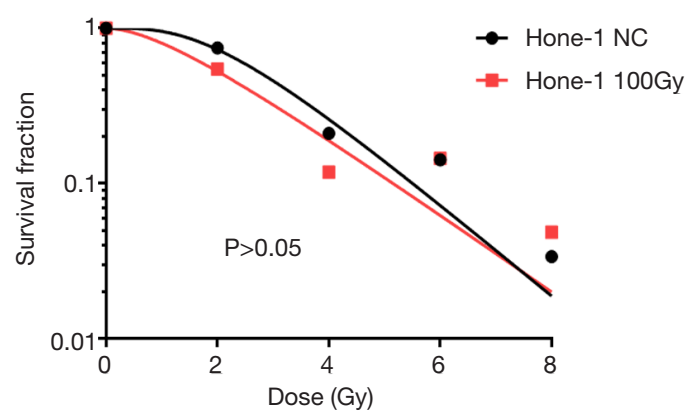

$\mathrm{E}$

Figure 1 A radioresistant NPC cell line, Hone-1R, was established. (A) Outline diagram summarizing the procedure of Hone-1R establishment. (B,C,D,E) Colony-forming assays were performed to detect the radioresistant capacity of Hone-1 cells after exposure to IR in a range of increasing doses. Hone-1 NC vs. Hone-1 60 Gy (B) Hone-1 NC vs. Hone-1 100 Gy (C) and 180 Gy (D,E). Photos were taken at $\times 10$ magnification; stained with $0.1 \%$ crystal violet $(D)$. Each data point represents the mean of three experiments \pm SD. NC, negative control. NPC, nasopharyngeal carcinoma.

L1 was obviously upregulated in Hone-1R cells, which was also indicated by immunofluorescence assay (Figure 2D). These findings demonstrated that radioresistant Hone$1 \mathrm{R}$ cells revealed an EMT-like phenotype with PD-L1 overexpression.

\section{Knockdown of PD-L1 reversed EMT and reduced migration ability of radioresistant Hone-1R cells}

To further examine the effects of PD-L1 on EMT and migration ability in NPC radioresistant Hone-1R cells,
PD-L1 was knocked down using siRNA in Hone-1R cells. The colony-forming assay showed that inhibition of PD-L1 expression led to a significant decrease in surviving clone numbers in Hone-1R cells exposed to different doses of IR $(2,4,6,8$, and $10 \mathrm{~Gy})(\mathrm{P}<0.01$, Figure $3 A, B)$, which revealed the role of PD-L1 in NPC radioresistance. Moreover, dramatic morphological changes after knockdown of PDL1 were observed, as shown in Figure 3C. Most of the Hone1R siPD-L1 cells lost mesenchymal cell shape and acquired tight cell-cell junctions compared with the Hone-1R si- 
A

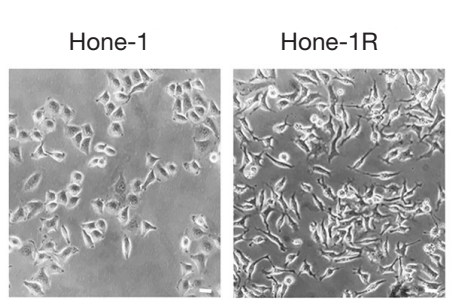

$\underline{D}$

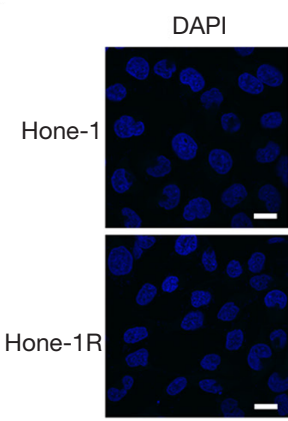

NC

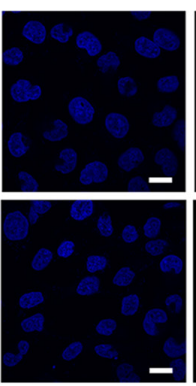

B
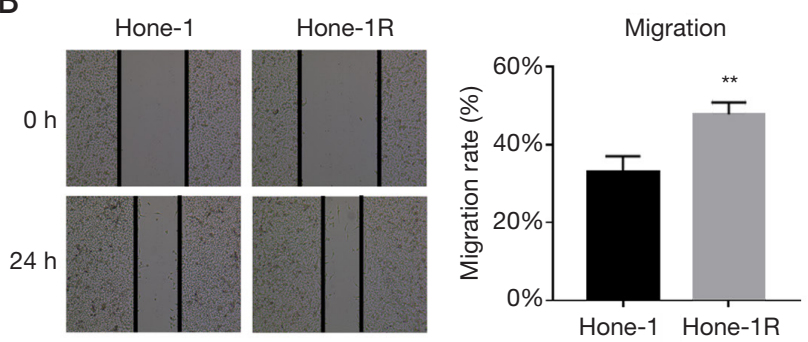

Merge

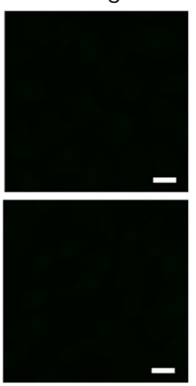

DAPI

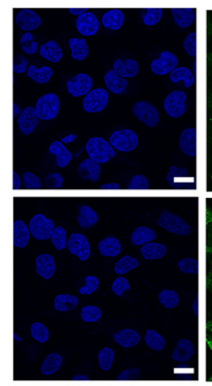

PD-L1

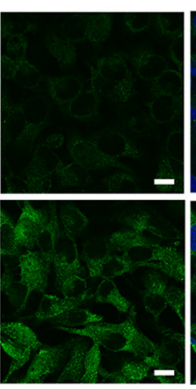

Merge

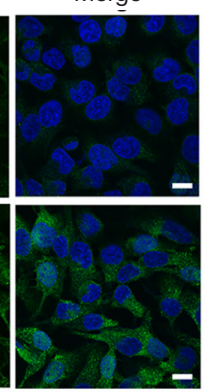

C

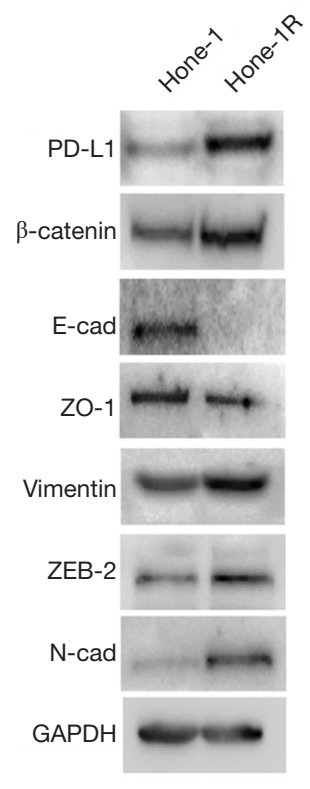

Figure 2 The radioresistant Hone-1R cell line presented an EMT-like phenotype with PD-L1 upregulation. (A) Photos were taken at 20x magnification. (B) A scratch wound healing assay was performed. Cell migration was photographed at 10x magnification at $0 \mathrm{~h}$ and $24 \mathrm{~h}$. Image J software (RRID: SCR_003070) was used to analyze the wound area and calculate the migration rates. The migration rate was calculated as described in "Methods". Bars, SD. **, P $<0.01$, Hone-1R vs. Hone-1. (C) EMT markers and PD-L1 were detected by Western blot (WB) analysis. (D) The cells were stained with an antibody to PD-L1 (green). Nuclei were stained with DAPI (4',6'-diamidino-2phenylindole). Images were captured by fluorescence microscopy at $\times 100$ magnification. Scale $=10 \mu \mathrm{m}$.

NC cells, which were similar in morphology to the Hone1 cells. Meanwhile, WB assay showed that knockdown of PD-L1 increased the expression of the epithelial marker E-cadherin, and concomitantly decreased the expression of the mesenchymal marker, vimentin. Additionally, knockdown of PD-L1 obviously inhibited the activation of ERK and STAT3 signaling pathways (Figure 3D). Furthermore, scratch wound healing assay also demonstrated that knockdown of PD-L1 by siRNA markedly inhibited migration of Hone-1R cells $(\mathrm{P}<0.05$, Figure $3 E, F)$. The above results demonstrated that $\mathrm{PD}-\mathrm{L} 1$ was involved in EMT and migration capacity in radioresistant Hone-1R cells.

\section{PD-L1 was overexpressed in higher stages and was associated with EMT status in NPC}

To further elucidate the role of PD-L1 in NPC, in silico analysis was performed based on public datasets of NPC. As described in "Methods", raw data from publicly available datasets focusing on the largest database of NPC patients were downloaded from GEO. As shown in Figure 4A, elevated expression levels of PD-L1 were detected in later-stage NPC patients (GSE103611). We performed GSVA to calculate the enrichment score of mesenchymal-phenotype gene signatures for each patient in GSE103611. Heatmap presented the correlation between PD-L1 and EMT-related genes. Relative PDL1 expression levels were compared by EMT spectrum (Figure 4B). We further calculated PD-L1-related EMT scores, and the results showed that PD-L1 mRNA expression was highly correlated with mesenchymal status $(\mathrm{P}=0.039$; Figure $4 C$ ). Additionally, using MSigDB hallmark database analysis, we identified that the top 10 significant pathways were closely related to the indicated immune response and EMT signaling pathways (Figure $4 D$ ). These results indicated that PD-L1 was overexpressed in the later stage and was associated with EMT status in NPC. 
A

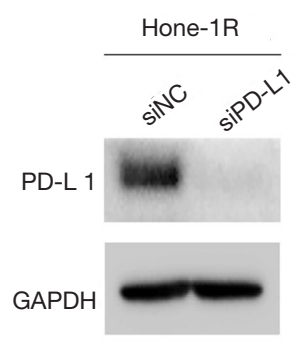

D

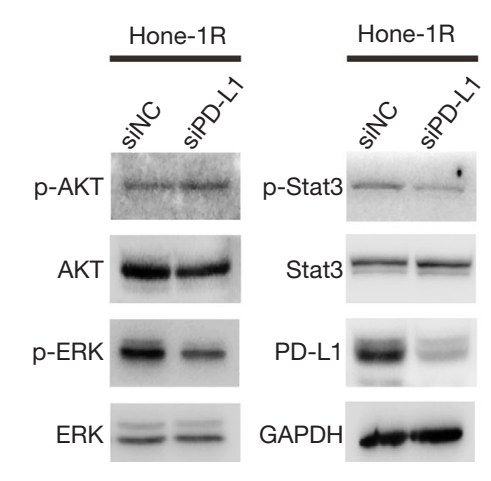

B

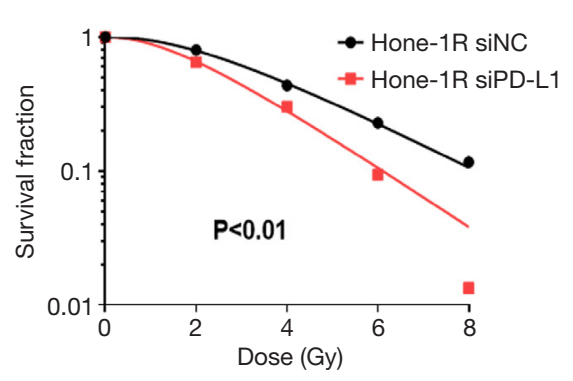

$\mathrm{E}$

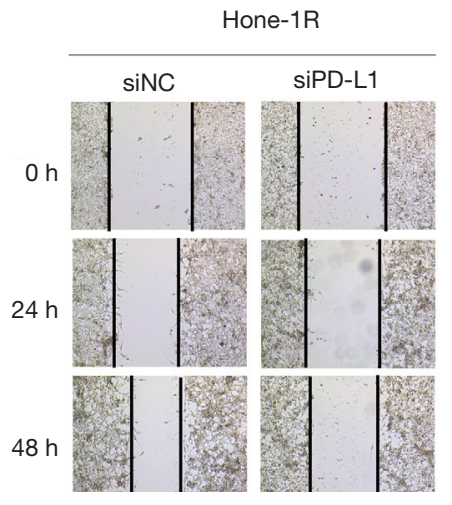

C

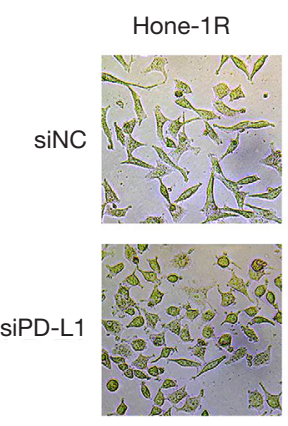

$\mathrm{F}$

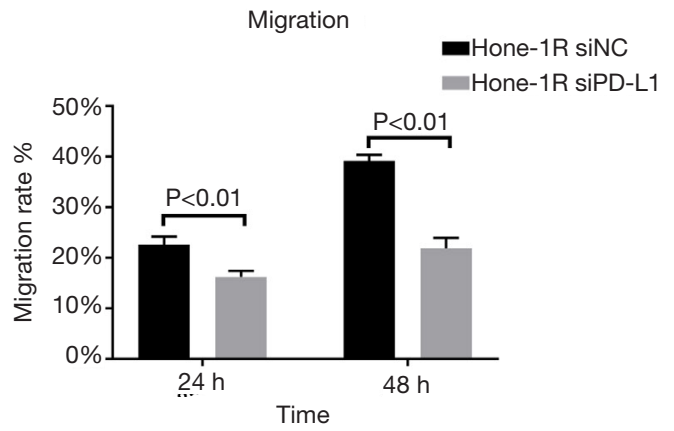

Figure 3 Knockdown of PD-L1 reversed EMT and reduced the migration ability of radioresistant Hone-1R cells. Knockdown of PD-L1 using siRNA in Hone-1R cells. (A) Cell lysates were collected for Western blot (WB) analysis after $48 \mathrm{~h}$ of siRNA transfection. (B) Colonyforming assay was performed to detect the radioresistant capacity of Hone-1R cells after exposure to IR in a range of increasing doses. (C) Photos were taken at 20× magnification after $48 \mathrm{~h}$ of siRNA transfection. (D) After $48 \mathrm{~h}$ of siRNA transfection, EMT markers and signaling pathways were detected by WB analysis. (E) Scratch wound healing assay was performed after $48 \mathrm{~h}$ of siRNA transfection. Cell migration was photographed at 10× magnification at 0,24 , and 48 h. (F) The migration rate was calculated as described in "Methods". Bars, SD. Hone$1 \mathrm{R}$ siNC $v$. Hone-1R siPD-L1. siNC, negative control siRNA.

\section{Discussion}

Radioresistance is a fundamental problem in tumor metastasis treatment and remains a challenging obstacle in NPC clinical therapy (16). The acquisition of radioresistance is a complicated process, which includes both intrinsic and acquired radioresistance (17). Although progress has been made in previous studies, the exact molecular mechanisms underlying long-term IR exposureinduced radioresistance and metastasis have yet to be elucidated in NPC. In our study, we proved that PD-L1 overexpression was significantly related to EMT score and later stage in NPC patients; in vitro functional analysis further indicated that PD-L1 was involved in the EMT process and migration capacity promotion in the NPC radioresistant Hone-1R cell line.
The biological phenomenon of EMT involves epithelial cells losing their cell-cell junctions and acquiring mesenchymal cells features such as higher migration capacity (8). This process is paralleled by a change in some of the markers, including vimentin up-regulation and E-cadherin down-regulation $(8,18)$. Increasing evidence has suggested the potential role of the EMT process in radioresistance of various tumors, such as esophageal squamous carcinoma and cervical cancer $(10,19)$. Furthermore, IR-induced EMT has been reported to contribute to enhanced invasiveness, migration, and radioresistance in non-small cell lung cancer and breast cancer (20,21). ZEB1 and snail are known to play important roles in IR-induced EMT $(22,23)$. A number of signaling pathways have been implicated involved in IR-induced EMT, including TGF- $\beta$, IGF-IR, Wnt, Hedgehog, Notch 

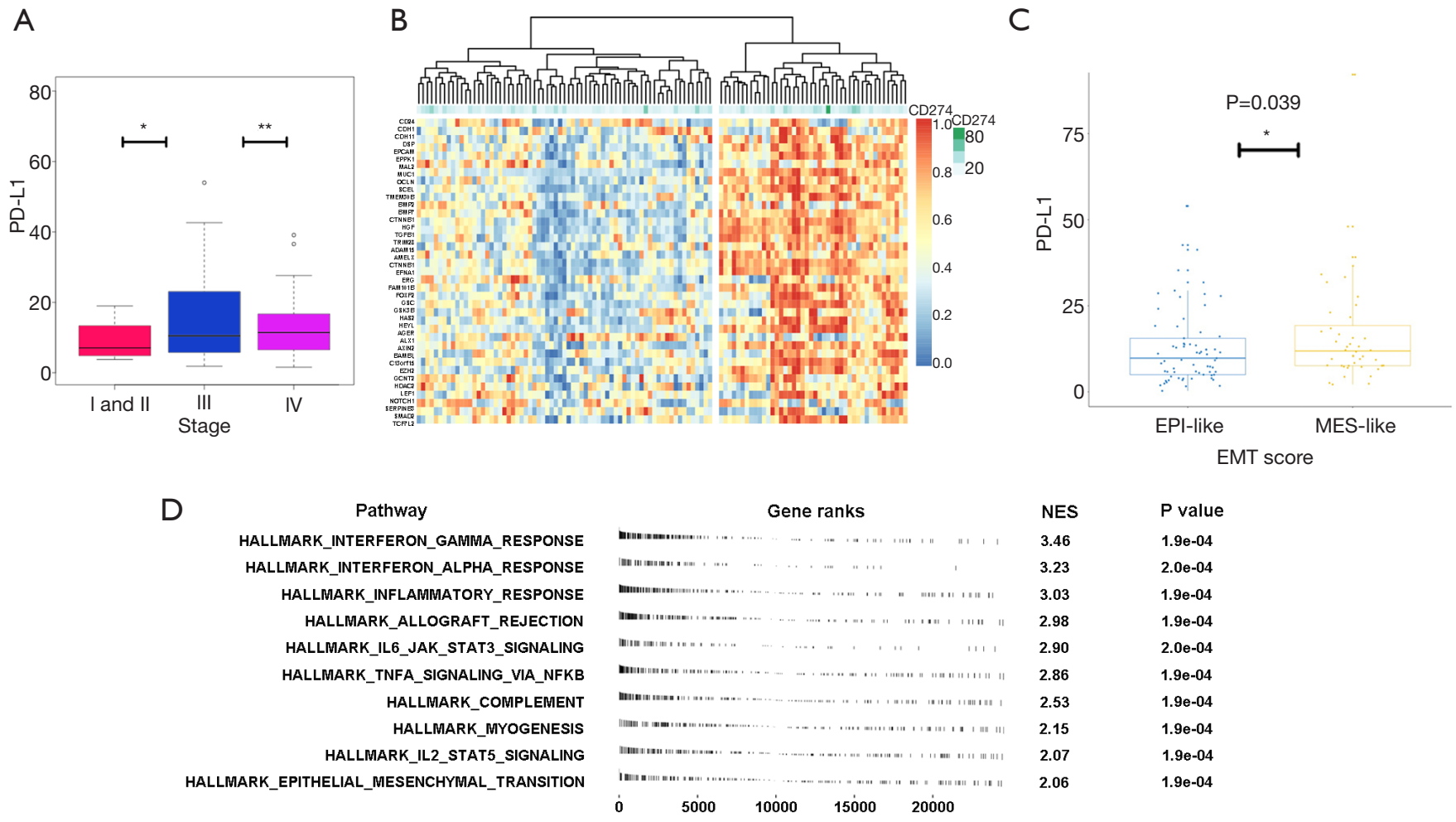

$\begin{array}{ll}\text { NES } & \text { P value } \\ 3.46 & 1.9 \mathrm{e}-04 \\ 3.23 & 2.0 \mathrm{e}-04 \\ 3.03 & 1.9 \mathrm{e}-04 \\ 2.98 & 1.9 \mathrm{e}-04 \\ 2.90 & 2.0 \mathrm{e}-04 \\ 2.86 & 1.9 \mathrm{e}-04 \\ 2.53 & 1.9 \mathrm{e}-04 \\ 2.15 & 1.9 \mathrm{e}-04 \\ 2.07 & 1.9 \mathrm{e}-04 \\ 2.06 & 1.9 \mathrm{e}-04\end{array}$

Figure 4 Later-stage disease showed PD-L1 overexpression, which correlated with EMT status in NPC patients. (A) Elevated expression levels of PD-L1 were detected in later-stage NPC patients (GSE103611). (B) The Heatmap represents the correlation between PD-L1 and EMT-related genes. (C) The correlation between expression levels of PD-L1 and EMT scores was analyzed. (D) Gene categories were enriched for genes revealed by MSigDB hallmark database analysis, owing to higher expression of PD-L1 in the GSE103611. The rows indicated the top 10 biological themes according to $\mathrm{P}$ values. NPC, nasopharyngeal carcinoma. * $\mathrm{P}<0.05 ;{ }^{* *}, \mathrm{P}<0.01$.

and PI3K/Akt (24-29). However, it remains unclear whether the PD-L1 molecule itself plays a role in the EMT process or if it has a general promotion effect on the migration and radioresistance abilities of NPC cells.

As a $\mathrm{T}$ cell inhibitory molecule, PD-L1 has been reported not only regulate $\mathrm{T}$ cell immunity but also to correlate with tumor progression and metastasis (30). Accumulating evidence on NPC has revealed that PD-L1 overexpression is associated with poor prognosis and may be a potential prognostic factor in NPC $(11,31)$. However, the molecular mechanism of PD-L1 in radioresistance and metastasis needs further investigation. In this study, we firstly established a radioresistant NPC cell, Hone-1R, and determined that PD-L1 was involved in long-term IRinduced EMT and migration in the Hone-1R cell line. Furthermore, based on in silico analysis of public datasets of NPC, it was demonstrated that PD-L1 was up-regulated in higher stage NPC patients and the expression level of
PD-L1 was highly correlated with mesenchymal status. Thus, PD-L1 was overexpressed in the later stage and was associated with EMT status in NPC. Upon MSigDB hallmark database analysis, we identified that the top 10 significant pathways were closely related to EMT signaling pathways. Therefore, there seems to be a close relationship between PD-L1-regulated migration ability and radioresistance exposure by long-term IR treatment in NPC cells.

\section{Conclusions}

In conclusion, our study elucidated the role of PD-L1 in long-term IR exposure-triggered EMT and radioresistance in NPC cells. Thus, targeting PD-L1 expression would inhibit migration and increase radiosensitivity in this type of NPC cell. Hence, patients with radioresistant NPC would likely benefit from anti-PD-1/PD-L1 therapy, and this avenue of treatment warrants further investigation. 


\section{Acknowledgments}

Funding: The study was supported by grants from Dalian Science and Technology Innovation Fund Project (Grant 2018J12SN063); Natural Science Foundation of Liaoning Province (Grant 2019-MS-010); Dalian Medical and Science Research Fund Project (Grant 1911096); Chinese Society of Clinical Oncology Research Funding (Grant Y-HR2018-117).

\section{Footnote}

Reporting Checklist: The authors have completed the MDAR checklist. Available at http://dx.doi.org/10.21037/tcr-201899

Data Sharing Statement: Available at http://dx.doi. org/10.21037/tcr-20-1899

Conflicts of Interest: All authors have completed the ICMJE uniform disclosure form (available at http://dx.doi. org/10.21037/tcr-20-1899). The authors have no conflicts of interest to declare.

Ethical Statement: The authors are accountable for all aspects of the work in ensuring that questions related to the accuracy or integrity of any part of the work are appropriately investigated and resolved. The study was conducted in accordance with the Declaration of Helsinki (as revised in 2013).

Open Access Statement: This is an Open Access article distributed in accordance with the Creative Commons Attribution-NonCommercial-NoDerivs 4.0 International License (CC BY-NC-ND 4.0), which permits the noncommercial replication and distribution of the article with the strict proviso that no changes or edits are made and the original work is properly cited (including links to both the formal publication through the relevant DOI and the license). See: https://creativecommons.org/licenses/by-nc-nd/4.0/.

\section{References}

1. Siegel RL, Miller KD, Jemal A. Cancer statistics, 2019. CA Cancer J Clin 2019;69:7-34.

2. Blanchard P, Lee A, Marguet S, et al. Chemotherapy and radiotherapy in nasopharyngeal carcinoma: an update of the MAC-NPC meta-analysis. Lancet Oncol
2015;16:645-55.

3. Lee AW, Ma BBY, Ng WT, et al. Management of nasopharyngeal carcinoma: current practice and future perspective. J Clin Oncol 2015;33:3356-64.

4. Xiao C, Wang L, Jiao Y, et al. Long-term results of concurrent chemoradiotherapy for T3/T4 locally advanced nasopharyngeal carcinoma. Mol Clin Oncol 2013;1:507-10.

5. Ng WT, Lee MC, Hung WM, et al. Clinical outcomes and patterns of failure after intensity-modulated radiotherapy for nasopharyngeal carcinoma. Int J Radiat Oncol Biol Phys 2011;79:420-8.

6. Blyth BJ, Cole AJ, MacManus MP, et al. Radiation therapy-induced metastasis: radiobiology and clinical implications. Clin Exp Metastasis 2018;35:223-36.

7. Sundahl N, Duprez F, Ost P, et al. Effects of radiation on the metastatic process. Mol Med 2018;24:16.

8. Thiery JP. Epithelial-mesenchymal transitions in tumour progression. Nat Rev Cancer 2002;2:442-54.

9. Tripathy J, Chowdhury AR, Prusty M, et al. $\alpha$-Lipoic acid prevents the ionizing radiation-induced epithelialmesenchymal transition and enhances the radiosensitivity in breast cancer cells. Eur J Pharmacol 2020;871:172938.

10. Yan S, Wang Y, Yang Q, et al. Low-dose radiation-induced epithelial-mesenchymal transition through NF- $\mathrm{\kappa B}$ in cervical cancer cells. Int J Oncol 2013;42:1801-6.

11. Huang ZL, Liu S, Wang GN, et al. The prognostic significance of PD-L1 and PD-1 expression in patients with nasopharyngeal carcinoma: a systematic review and meta-analysis. Cancer Cell Int 2019;19:141.

12. Liu $X$, Shan C, Song Y, et al. Prognostic value of programmed cell death ligand-1 expression in nasopharyngeal carcinoma: a meta-analysis of 1315 patients. Front Oncol 2019;9:1111.

13. Keir ME, Butte MJ, Freeman GJ, et al. PD-1 and its ligands in tolerance and immunity. Annu Rev Immunol 2008;26:677-704.

14. Fei Z, Deng Z, Zhou L, et al. PD-L1 Induces EpithelialMesenchymal Transition in Nasopharyngeal Carcinoma Cells Through Activation of the PI3K/AKT Pathway. Oncol Res 2019;27:801-7.

15. Grada A, Otero-Vinas M, Prieto-Castrillo F, et al. Research Techniques Made Simple: Analysis of Collective Cell Migration Using the Wound Healing Assay. J Invest Dermatol 2017;137:e11-e16.

16. Lu H, Yao M. The current status of intensity-modulated radiation therapy in the treatment of nasopharyngeal carcinoma. Cancer Treat Rev 2008;34:27-36. 
17. Shimura T. Targeting the AKT/cyclin D1 pathway to overcome intrinsic and acquired radioresistance of tumors for effective radiotherapy. Int J Radiat Biol 2017;93:381-5.

18. Heerboth S, Housman G, Leary M, et al. EMT and tumor metastasis. Clin Transl Med 2015;4:6.

19. Zang C, Liu X, Li B, et al. IL-6/STAT3/TWIST inhibition reverses ionizing radiation-induced EMT and radioresistance in esophageal squamous carcinoma. Oncotarget 2017;8:11228.

20. Choi YJ, Baek GY, Park HR, et al. Smad2/3-regulated expression of DLX2 is associated with radiation-induced epithelial-mesenchymal transition and radioresistance of A549 and MDA-MB-231 human cancer cell lines. PLoS One 2016;11:e0147343.

21. Jung JW, Hwang SY, Hwang JS, et al. Ionising radiation induces changes associated with epithelial-mesenchymal transdifferentiation and increased cell motility of A549 lung epithelial cells. Eur J Cancer 2007;43:1214-24.

22. Chen $W, W u$ S, Zhang G, et al. Effect of AKT inhibition on epithelial-mesenchymal transition and ZEB1potentiated radiotherapy in nasopharyngeal carcinoma. Oncol Lett 2013;6:1234-40.

23. Mahabir R, Tanino M, Elmansuri A, et al. Sustained elevation of Snail promotes glial-mesenchymal transition after irradiation in malignant glioma. Neuro Oncol 2014;16:671-85.

24. Martin M, Vozenin MC, Gault N, et al. Coactivation of AP-1 activity and TGF-beta 1 gene expression in the stress response of normal skin cells to ionizing radiation.

Cite this article as: Li H, Wang Z, Liang S, Liu Q, Wang P, Cai L, Wang R. Radiation induces epithelial to mesenchymal transition via upregulation of PD-L1 in nasopharyngeal carcinoma cell. Transl Cancer Res 2021;10(1):372-381. doi: $10.21037 /$ tcr-20-1899
Oncogene 1997;15:981-9.

25. Wang Z, Liu G, Mao J, et al. IGF-1R Inhibition Suppresses Cell Proliferation and Increases Radiosensitivity in Nasopharyngeal Carcinoma Cells. Mediators Inflamm 2019;2019:5497467.

26. Bastos LG, de Marcondes PG, de-Freitas-Junior JC, et al. Progeny from irradiated colorectal cancer cells acquire an EMT-like phenotype and activate $W \mathrm{nt} / \beta$-catenin pathway. J Cell Biochem 2014;115:2175-87.

27. Gan GN, Eagles J, Keysar SB, et al. Hedgehog signaling drives radioresistance and stroma-driven tumor repopulation in head and neck squamous cancers. Cancer Res 2014;74:7024-36.

28. Kim RK, Kaushik N, Suh Y, et al. Radiation driven epithelial-mesenchymal transition is mediated by Notch signaling in breast cancer. Oncotarget 2016;7:53430-42.

29. He E, Pan F, Li G, et al. Fractionated Ionizing Radiation Promotes Epithelial-Mesenchymal Transition in Human Esophageal Cancer Cells through PTEN DeficiencyMediated Akt Activation. PLoS One 2015;10:e0126149.

30. Gong X, Li X, Jiang T, et al. Combined radiotherapy and anti-PD-L1 antibody synergistically enhances antitumor effect in non-small cell lung cancer. J Thorac Oncol 2017;12:1085-97.

31. Zhang M, Wei S, Su L, et al. Prognostic significance of pretreated serum lactate dehydrogenase level in nasopharyngeal carcinoma among Chinese population: A meta-analysis. Medicine (Baltimore) 2016;95:e4494. 